\title{
ROLE OF PALLIATION IN STAGE IV CARCINOMA CERVIX
}

Smriti Chachan, C. K. Bose, A. Mukhopadhyay

1. Resident Medical Officer. Preventive Oncology Unit, Netaji Subhas Chandra Bose Cancer Research Institute, Kolkata, India.

2. Consultant. Surgical Oncology Unit, Netaji Subhas Chandra Bose Cancer Research Institute, Kolkata, India.

3. Director \& Head. Medical Oncology Unit, Netaji Subhas Chandra Bose Cancer Research Institute, Kolkata, India.

\section{CORRESPONDING AUTHOR:}

Dr. Smriti Chachan, 14A Russa Road East 2nd Lane,

Swiss Park, Kolkata-700033.

E-mail: smriti_chachan@yahoo.co.in

Ph: 00919903543769

ABSTRACT: BACKGROUND: Palliation reduces the severity of disease symptoms, rather than reversing its progression or providing a cure. Metastatic cancer cervix $(\mathrm{Ca} \mathrm{Cx})$ is incurable by surgery, radiation or chemotherapy, but these modalities are useful for palliation. Globally about five to six lakh new cases of carcinoma cervix are diagnosed every year. Of these, one lakh cases are diagnosed in India of which $25.0 \%$ are from West Bengal only. OBJECTIVES: Our objective was to study the role of palliation in Stage IV Carcinoma Cervix. SETTINGS AND DESIGN: During the study period of five years from January 2007 to December 2011, consecutive seventy five new cases of stage IV carcinoma cervix diagnosed at Netaji Subhas Chandra Bose Cancer Research Institute, Kolkata, were included in our study. MATERIALS AND METHODS: Clinical examination with relevant investigations like kidney function tests (KFT), biopsy, cystoscopy, CT scan etc were done for diagnosis \& staging. Treatment was decided based on woman's age, general health and the location \& type of the tumour. Treatment options were surgery, radiotherapy (RT), chemotherapy (CT) and simple palliation. In our study, combined CT+RT was done in $18.67 \%$ patients most of who presented with Stage IV disease. Radiation was given as brachytherapy following teletherapy. Chemotherapy was used as adjunct to RT or for palliation or as neo-adjuvant chemotherapy (NACT), most commonly using paclitaxel (135mg/square metre), cisplatin (50mg/ square metre) and 5- fluorouracil (600mg/ square metre). At times, chemotherapy could provide pain relief only. Vault smear and metastatic workup was done during follow-up visits every 8-12 weeks after treatment completion. RESULTS: Majority of patients belonged to the age group 42-69 years with a median age of 53 years. Bladder involvement was seen in 15(20.0\%) cases, bowel involvement in $14(19.0 \%)$ and distant metastasis in $46(61.0 \%)$ cases. Most cases were of Squamous cell carcinoma $(94.0 \%)$, clinically presenting with foul smelling vaginal discharge \pm bleeding per vaginum (100.0\%). In our study, significant amount (30.0\%) of disease control for a substantial period (1.5 years) was observed. The overall survival rate in patients of Stage IV carcinoma cervix on various forms of palliative treatment was 18.0\%. CONCLUSION: Palliation is achieved by surgery, radiation or chemotherapy, though at times, simple palliation is the only feasible option. The poor survival rate was probably due to improper reporting \& lack of regular follow up in the given socio-economic background. As cure cannot be achieved in advanced carcinoma cervix, palliation is important in alleviating the distressing symptoms and improving the quality of life. 
KEY WORDS: Palliation, carcinoma cervix, metastatic, radiation, brachytherapy, teletherapy, chemotherapy, neo-adjuvant chemotherapy.

INTRODUCTION: Cervical cancer is a major health problem for women. It is the third most common cancer worldwide. [1,2] Globally about 5-6 lakh new cases of carcinoma cervix (Ca Cx) are diagnosed every year. Of these 1 lakh cases are diagnosed in India. One quarter of this disease burden is shared by West Bengal. Persistent Human Papilloma Virus (HPV) infection is regarded as the most important factor contributing to the development of $\mathrm{Ca} \mathrm{Cx}$. ${ }^{[2]}$ Immunization against HPV prevents infection with certain types of HPV (type 6,11,16,18 mainly) \& is expected to prevent Ca Cx. ${ }^{[4-8]}$ Type 16 is the most commonly implicated serotype of HPV for this. Other epidemiological risk factors associated with Ca Cx are history of smoking, parity, contraceptive use, early age of onset of coitus, multiple partners, history of sexually transmitted disease \& chronic immunosuppression. [9]

During the past two decades, increasing attention has been paid to quality-of-life issues in oncology. ${ }^{[4,10]}$ As the hospice movement has grown in the country, palliative care has developed into an integral part of (rather than the antithesis of ) comprehensive cancer care. [4] Most patients who receive the hospice care are referred too late for adequate benefit or never referred at all. Effective palliative care needs an interdisciplinary team approach.

By definition palliation, derived from Latin word palliare, is any form of treatment that concentrates on reducing the severity of disease symptoms, rather than striving to halt, delay, or reverse progression of the disease itself or, provide a cure. The goal of palliative care is to prevent and relieve suffering and to support the best possible quality of life for patients and their families, regardless of the stage of the disease or the need for other therapies. ${ }^{3]}$ Metastatic cancer cervix cannot be cured by surgery, radiation or chemotherapy, but these modalities are useful for palliation. Recurrence is also an indication for palliation.

Our objective was to study the role of palliation in patients of Stage IV Ca Cx.

MATERIALS AND METHODS: During the study period of five years (January 2007 to December 2011), consecutive 75 new cases of stage IV Ca Cx diagnosed at Netaji Subhas Chandra Bose Cancer Research Institute, Kolkata, were included in our study. Staging was done as per FIGO classification for cancer cervix (Table 1).

The study was approved by our Institutional Ethics Committee. Clinical examination with relevant investigations like chest X-ray (CXR), ultrasonography (USG), kidney function tests (KFT), biopsy, cystoscopy, CT scan etc. were done for diagnosis \& staging which were tallied with the National Comprehensive Cancer Network (NCCN) 2011 guidelines later. Treatment was decided based on woman's age, general health (as per ECOG scale) and the location \& type of the tumour. Treatment options were Surgery, RT, CT and simple palliation in the form of best supportive care possible. Radiation was given as teletherapy (total dose of 60 Gy usually @ 1-1.8 Gy/fraction) followed by brachytherapy. Chemotherapy was used as adjunct to RT or for palliation. At times, neo-adjuvant chemotherapy (NACT) was given in patients with bulky disease, locally advanced cancers etc. Paclitaxel (135 mg/sq.m), cisplatin (50 mg/sq.m) and 5- fluorouracil $(600 \mathrm{mg} / \mathrm{sq} . \mathrm{m})$ were the most commonly used chemotherapeutic agents. Cisplatin was used for concurrent chemotherapy with RT as single agent. However, it was used in combination with the other agents as well without significantly comparable results.

Vault smear and metastatic workup was done during follow-up of these patients every 8-12 weeks after completion of treatment. 
RESULTS: Our youngest patient was 21 yrs old and the eldest lady was 79 yrs of age. Majority of patients belonged to the age group 42-69 years with a median age of 53 years (Table 2).

Bladder involvement was seen in 15 (20.0\%), bowel involvement in $14(19.0 \%)$ and distant metastasis in $46(61.0 \%)$ cases. Histologically, grade III Ca Cx was the most common presentation (Figure 1). Most cases were of Squamous cell Ca (94.0\%) (Figure 2). The most common clinical presentation was foul smelling vaginal discharge \pm bleeding per vaginum (100.0\%) (Figure 3).

In our study, combined CT+RT was done in $18.67 \%$ patients most of who presented with Stage IV disease (Table 3). However, on few occasions, chemotherapy could

provide pain relief only. Significant amount (30.0\%) of disease control for a substantial period (1 year 6 months) was observed. The survival rate in patients of Stage IV Ca Cx who were given various forms of palliative treatment was found to be $18.0 \%$.

DISCUSSION: Ca $\mathrm{Cx}$ continues to be the most common malignancy with a compromised quality of life especially in the developing world. Though incidence of $\mathrm{Ca} \mathrm{Cx}$ is on the rise, it is expected the introduction of measures like HPV vaccines to go a long way to control the rising trend. HPV vaccine has shown promising results in the short phase of study as per national health surveys.[11]

The palliative care guidelines are the first NCCN guidelines to include death as an expected outcome and after-death care for the family as an essential part of the continuum of cancer care. All cancer patients should be screened for palliative care needs at their initial visit, at appropriate intervals and as clinically indicated. [3] Educational programs should be provided to all healthcare professionals and trainees so that they can develop effective palliative care knowledge, skills and attitudes.

Criteria for early consultation with a palliative care specialist are based on patient characteristics, social circumstances and anticipatory bereavement issues.

In the absence of any clear cut indications for palliative care, advanced disease, aggressive disease histology, poor general condition of the patient, presence of distant metastasis, social stigma, toxicity of therapy as compared to the benefit and the treating oncologist's discretion are the main indications for which palliation is used.

Concurrent chemoradiation, using cisplatin-based chemotherapy, is the treatment of choice for stages IB2, II, III and IV A disease based on the results of five randomised clinical trials. ${ }^{[3,12,13]}$

CONCLUSION: Palliation is achieved by surgery, radiation or chemotherapy, however, at times, simple palliation in the form of best supportive care is the only feasible option. [14-16] A good clinical history and examination is good enough for a clinical diagnosis of advanced $\mathrm{Ca} C \mathrm{Cx}$, specially in a setting without adequate facilities like rural Bengal which accounts for a significant proportion of the disease burden. The poor survival rate in our study was probably due to improper reporting and lack of regular follow up in the given socio-economic background. As cure cannot be achieved in advanced $\mathrm{Ca} \mathrm{Cx}$, palliation has a great role to play in alleviating the distressing symptoms and improving the quality of life.

\section{REFERENCES:}

1. Parkin DM, Bray F, Ferlay J, Pisani P. Global cancer statistics 2002. CA Cancer J Clin 2005; 55(2): 74-108. 
Available at: http:://www.ncbi.nlm.nih.gov/pubmed/15761078.

2. Kamangar F, Dores GM, Anderson WF. Patterns of cancer incidence, mortality \& prevalence across 5 continents: defining priorities to reduce cancer disparities in different geographic regions of the world. J Clin Oncol 2006; 24: 2137-2150. Available at: http://www.ncbi.nlm.nih.gov/pubmed/16682732.

3. NCCN guidelines for cervical cancer 2011. Available at: http://www.nccn.org/professionals/physician_gls/f_guidelines.asp.

4. Villa LL, Costa RL, Petta CA et al. Prophylactic quadrivalent human papilloma virus (types 6,11,16,18) L1 virus -like particle in young women: a randomised double-blind placebo-controlled multicentre phase II efficacy trial. Lancet Oncol 2005; 6: 271-278. Available at: http://www.ncbi.nlm.nih.gov/pubmed/15863374.

5. Ault KA. Effect of prophylactic human papillomavirus L1 virus-like-particle vaccine on risk of CIN grade 2, grade 3 \& adenoca in situ: a combined analysis of 4 RCT. Lancet 2007; 369: 1861-1868.

Available at: http://www.ncbi.nlm.nih.gov/pubmed/17544766.

6. Quadrivalent vaccine against HPV to prevent high grade cervical lesions. N Engl J Med 2007; 356: 1915-1927.

Available at: http://www.ncbi.nlm.nih.gov/pubmed/17494925.

7. Arbyn M, Dillner J. Review of current knowledge on HPV vaccination: an appendix to the European Guidelines for quality Assurance in Ca Cx screening. J Clin Virol 2007; 38: 189197.

Available at: http://www.ncbi.nlm.nih.gov/pubmed/17258503.

8. Rambout L, Hopkins L, Hutton B, Fergusson D. prophylactic vaccination against HPV infection \& disease in women: a systematic review of RCTs. CMAJ 2007;177: 469-479. Available at: http://www.ncbi.nlm.nih.gov/pubmed/17671238.

9. Comparison of risk factors for invasive squamous cell ca \& adenoca of cx: collaborative reanalysis of individual data on 8097 women with SCC \& 1734 women with adenoca from 12 epidemiological studies. Int J Cancer 2007; 120: 885-891. Available at: http://www.ncbi.nlm.nih.gov/pubmed/17131323.

10. ASCO task force for cancer care at the end of life. Cancer care during the last phase of life. J Clin Oncol. 1998; 16: 1986-1996.

11. HPV Vaccine Information for Clinicians - Fact Sheet. Available at: http://www.cdc.gov/std/hpv/stdfact-hpv-vaccine-hcp.htm

12. Morris M, Eifel PJ, Lu J et al. Pelvic radiation with concurrent chemotherapy compared with pelvic \& para-aortic radiation for high risk cervical cancer. N Engl J Med 1999; 340: 1154-1161.

13. Peters WA, Liu PY, Barrett RJ et al. Concurrent chemotherapy and pelvic radiation compared with pelvic radiation alone as adjuvant after radical surgery in high risk earlystage Ca Cx. J Clin Oncol 2000;18:1606-1613.

Available at: http://www.ncbi.nlm.nih.gov/pubmed/10764420.

14. Dahistrom LA, Ylitalo N, Sundstorm K et al. Prospective study of HPV and risk of cervical adenocarcinoma. Int J Cancer 2010; 127: 1923-1930.

Available at: http://www.ncbi.nlm.nih.gov/pubmed/20473898.

15. Ramirez PT, Slomovitz BM, Soliman PT et al. Total laparoscopic radical hysterectomy \& lymphadenectomy: the M D Anderson Cancer Centre experience. Gyne Oncol 2006; 102: 252-255. 
Available at: http://www.ncbi.nlm.nih.gov/pubmed/16472844.

16. Lowe MP, Chamberlain DH, Kamelle SA et al. A multi-institutional experience with robotic assisted radical hysterectomy for early stage Ca Cx. Gyne Oncol 2009; 113: 191194. Available at: http://www.ncbi.nlm.nih.gov/pubmed/19249082.

Table 1: International Federation of Gynaecology and Obstetrics (FIG0) staging of carcinoma cervix

\begin{tabular}{|c|c|c|}
\hline Stages & Subdivision & Description \\
\hline \multirow[t]{4}{*}{ I } & I A1 & $\begin{array}{l}\text { Invasive carcinoma with stromal invasion } \leq 3 \mathrm{~mm} \text { in depth and } \leq 7 \\
\mathrm{~mm} \text { in horizontal spread }\end{array}$ \\
\hline & I A2 & Stromal invasion 3-5 $\mathrm{mm}$ in depth with horizontal spread $\leq 7 \mathrm{~mm}$ \\
\hline & I B1 & Clinically visible lesion $\leq 4 \mathrm{~cm}$ in greatest dimension. \\
\hline & I B2 & Clinically visible lesion $\geq 4 \mathrm{~cm}$ in greatest dimension. \\
\hline \multirow[t]{2}{*}{ II } & II A & Tumor without parametrial invasion \\
\hline & II B & Tumor with parametrial invasion \\
\hline \multirow[t]{2}{*}{ III } & III A & Tumor involves lower third of vagina, no extension to pelvic wall \\
\hline & III B & $\begin{array}{l}\text { Tumor extends to pelvic wall } \pm \text { hydronephrosis or non-functioning } \\
\text { kidney }\end{array}$ \\
\hline \multirow[t]{2}{*}{ IV } & IV A & $\begin{array}{l}\text { Tumor invades mucosa of bladder or rectum } \pm \text { extends beyond } \\
\text { pelvis }\end{array}$ \\
\hline & IV B & Distant metastases \\
\hline
\end{tabular}

Table 2: Age distribution ( $n=75)$

\begin{tabular}{ccc}
\hline Age Distribution (yrs) & Number of women & Percentage (\%) \\
\hline $21-30$ & 3 & 4 \\
$31-40$ & 8 & 11 \\
$41-50$ & 16 & 21 \\
$51-60$ & 33 & 44 \\
$61-70$ & 13 & 17 \\
$71-80$ & 2 & 3 \\
\hline
\end{tabular}

Table 3: Primary treatment given to the patients $(n=75)$

\begin{tabular}{ccc}
\hline Treatment & Number of patients & Percentage (\%) \\
\hline Surgery & 2 & 2.7 \\
CT+RT(including NACT) & 14 & 18.67 \\
RT alone & 59 & 78.83 \\
\hline
\end{tabular}


Table 4: ECOG performance status

\section{Grade ECOG}

$0 \quad$ Fully active, able to carry on all pre-disease performance without restriction

1 Restricted in physically strenuous activity but ambulatory and able to carry out work of a light or sedentary nature, e.g., light house work, office work

2 Ambulatory and capable of all selfcare but unable to carry out any work activities. Up and about more than $50 \%$ of waking hours

3 Capable of only limited selfcare, confined to bed or chair more than $50 \%$ of waking hours

4 Completely disabled. Cannot carry on any selfcare. Totally confined to bed or chair

5 Dead

Figure 1: Grade of the tumour as per the histopathological examination (HPE) report

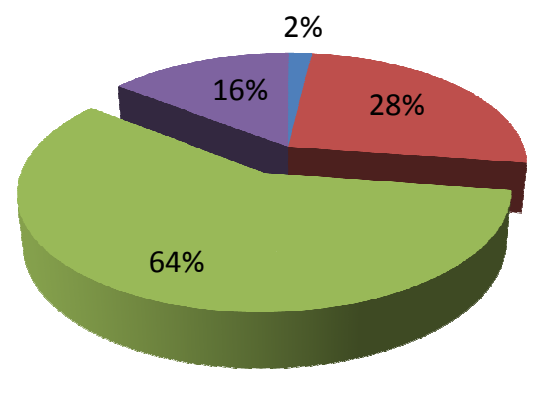

- GRADE I

- GRADE II

$\square$ GRADE III

NOT KNOWN

Figure 2: Histopathological distribution of patients

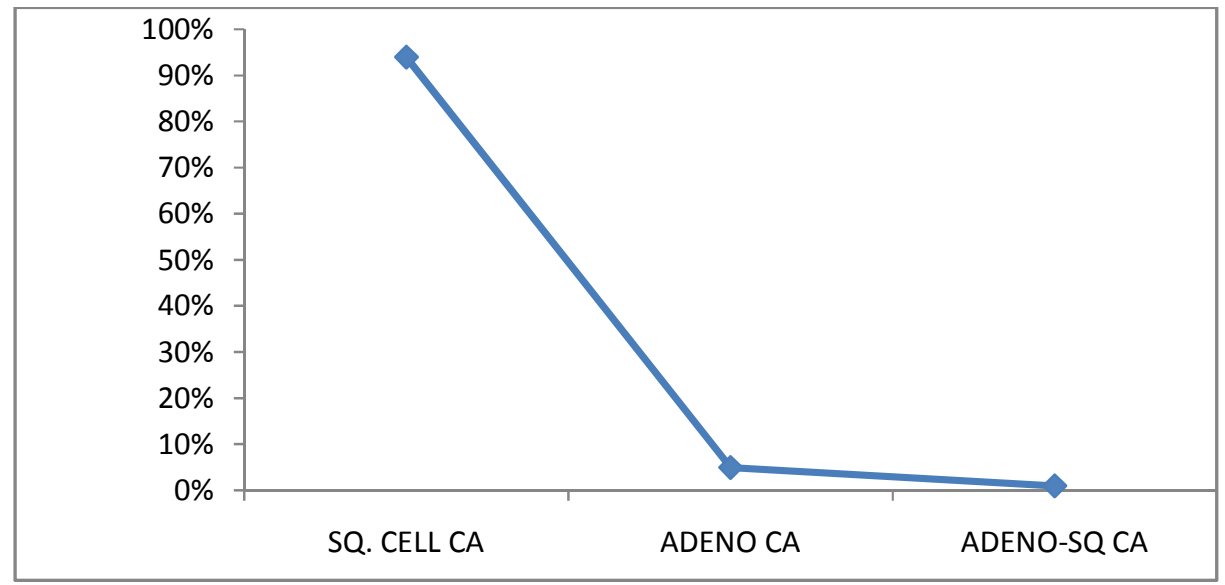




\section{ORIGINAL ARTICLE}

Figure 3: Symptomatology of the patients
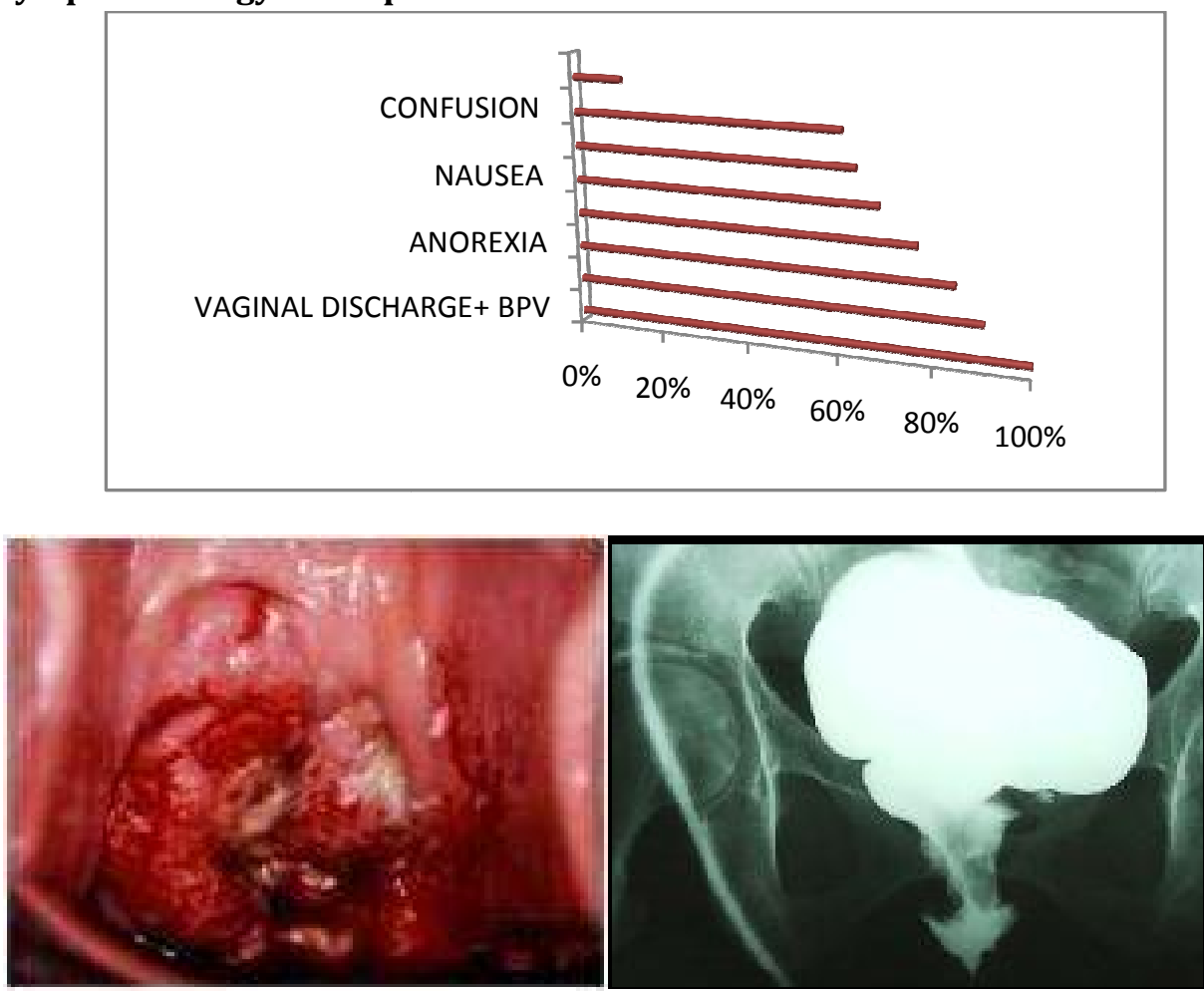

Image 1: Speculum view of advanced $\mathrm{Ca} \mathrm{Cx}$ in one of our patients

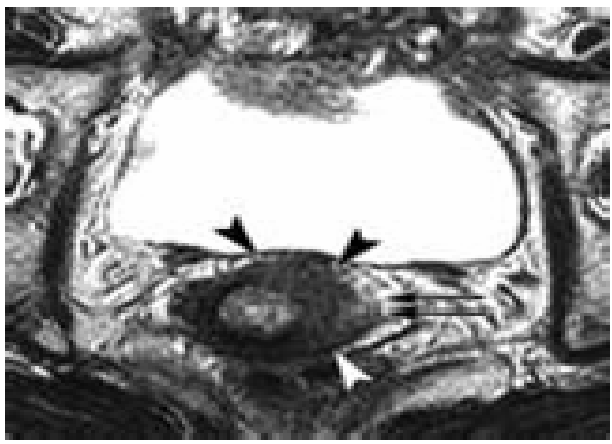

Image 3: Hysterosalpingography (HSG) showing a vesicovaginal fistula

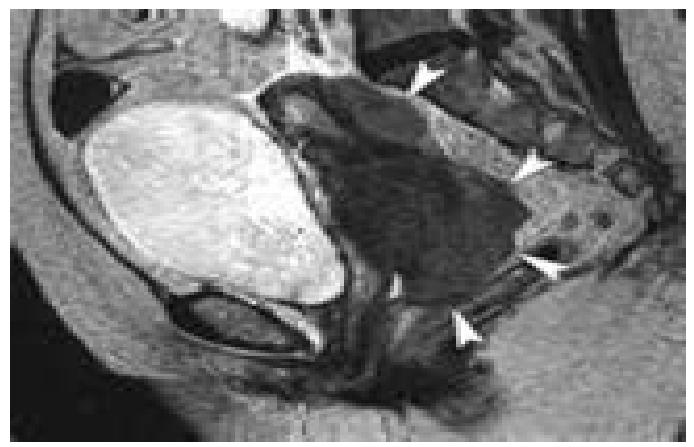

Images 2 (a) and (b):Urinary bladder infiltration as per MRI images in a_diagnosed case of Ca Cx.
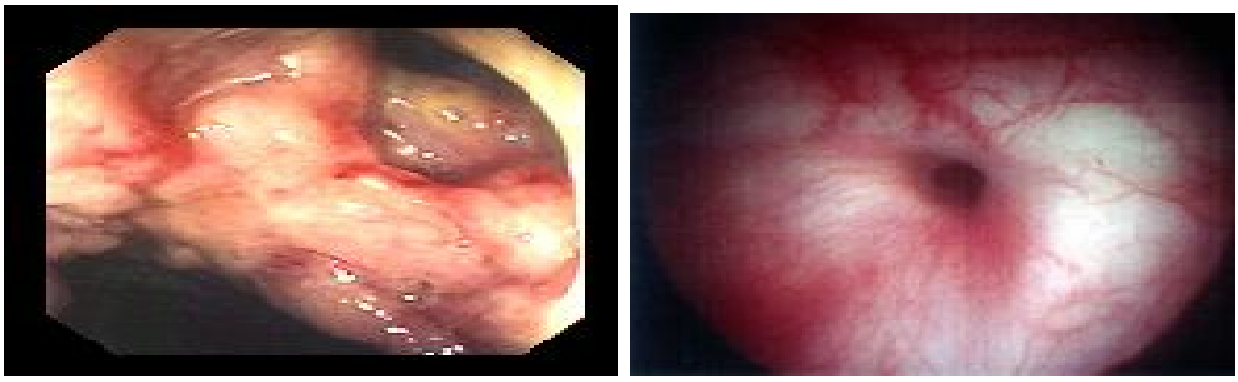

Image 4: Recto-Vaginal Fistula (RVF) Image 5: Cystoscopic view of Vesico-Vaginal Fistula (VVF) 